\title{
Microarray analysis in caudal medulla of cattle orally challenged with bovine spongiform encephalopathy
}

\author{
L.M. Almeida ${ }^{1,2}$, U. Basu ${ }^{1}$, J.L. Williams ${ }^{3}$, S.S. Moore ${ }^{1}$ and L.L. Guan ${ }^{1}$ \\ ${ }^{1}$ Department of Agricultural, Food and Nutritional Science, \\ University of Alberta, Edmonton, Canada \\ ${ }^{2}$ Universidade Estadual de Goiás, Ipameri, GO, Brasil \\ ${ }^{3}$ Parco Technologico Padano, Polo Universitario, Lodi, Italy \\ Corresponding author: L.M. Almeida \\ E-mail: almeidalm@hotmail.com
}

Genet. Mol. Res. 10 (4): 3948-3962 (2011)

Received August 8, 2011

Accepted September 13, 2011

Published October 25, 2011

DOI http://dx.doi.org/10.4238/2011.October.25.5

\begin{abstract}
Bovine spongiform encephalopathy (BSE) is a fatal disorder in cattle characterized by progressive neurodegeneration of the central nervous system. We investigated the molecular mechanisms involved in neurodegeneration during prion infection through the identification of genes that are differentially expressed (DE) between experimentally infected and non-challenged cattle. Gene expression of caudal medulla from control and orally infected animals was compared by microarray analysis using 24,000 bovine oligonucleotides representing 16,846 different genes to identify DE genes associated with BSE disease. In total, $182 \mathrm{DE}$ genes were identified between normal and BSE-infected tissues $(>2.0$-fold change, $\mathrm{P}<0.01)$; $81 \mathrm{DE}$ genes had gene ontology functions, which included synapse function, calcium ion regulation, immune and inflammatory response, apoptosis, and cytoskeleton organization; 13 of these genes were found to be involved in 26 different Kyoto Encyclopedia of Genes and Genomes (KEGG) pathways. The expression of five DE genes associated with synapse function (tachykinin, synuclein, neuropeptide Y, cocaine,
\end{abstract}


amphetamine-responsive transcript, and synaptosomal-associated protein $25 \mathrm{kDa}$ ) and three $\mathrm{DE}$ genes associated with calcium ion regulation (parvalbumin, visinin-like, and cadherin) was further validated in the medulla tissue of cattle at different infection times $(6$, 12,42 , and 45 months post-infection) by qRT-PCR. These data will contribute to a better understanding of the molecular mechanisms of neuropathology in bovine species.

Key words: Microarray; Transmissible spongiform encephalopathy; Prion ${ }^{\mathrm{BSE}}$; Orally challenged

\section{INTRODUCTION}

Bovine Spongiform Encephalopathy (BSE), an infectious neurodegenerative disease in cattle, is one of the fatal transmissible sponge encephalopathies (TSEs) that have been identified in many mammalian species including humans. These diseases are characterized by neurodegeneration and aggregation of aberrantly folded prion protein (Aguzzi et al., 2008). While the physiological changes associated with TSE disease in the brain are well documented, the underlying molecular events involved in neurodegeneration are poorly defined. Some studies have shown that the probability of infection and the outcome of the disease are genetically controlled (Prusiner and Scott, 1997). In sheep, mice and human, a large part of the natural susceptibility to TSE depends on alleles of the PRPN gene (Moreno et al., 2003). However, in cattle the association between Prpn variations and disease incidence is less well defined (Juling et al., 2006), which suggests that genes other than PRPN may be involved in susceptibility of cattle to BSE. Quantitative trait loci (QTL) studies have identified genomic regions associated with disease susceptibility, on Bos tauros chromosomes (BTA) 5, 10, and 20 (Hernandez-Sanches et al., 2002); BTA1, -6, -13, -17, -19 and -X (Zhang et al., 2004); and BTA2, -14, -16, -20, -21 and 28 (Murdoch et al., 2010).

In addition to QTL analysis, other approaches have been used to investigate the molecular mechanisms involved in neurodegeneration associated with prion diseases by identification of genes differentially expressed (DE) between normal and infected tissues These studies have detected differential gene expression by the analysis of cDNA libraries (Diedrich et al., 1991), mRNA differential display (Dandoy-Dron et al., 1998), suppression subtractive hybridization (Kopacek et al., 2000) and more recently using microarrays (Riemer et al., 2004; Greenwood et al., 2005; Sorensen et al., 2008). These studies have revealed multiple genes and signaling pathways that may be involved in TSE pathogenesis.

Recently, we reported 101 DE genes between normal and BSE-infected Peyer's patch tissues (Khaniya et al., 2009) and 966 DE genes in medulla (Almeida et al., 2011) in cattle orally infected with BSE agent 12 and 45 months post-infection. In the present study, a pooling approach was used in order to analyze a large number of animals and to decrease the individual variation. In total $182 \mathrm{DE}$ genes were identified, from which $45 \%$ had previously been detected in a comparison between controls and both 12- and 45-month post-infection animals. Differences in gene expression associated with synapse function and calcium ion regulation were validated by qRT-PCR. Global gene expression analyses through identification of genes DE in response to BSE will help to understand the disease process and may result 
in the discovery of biomarkers for disease progression, therapeutic targets and elucidate the mechanisms of neuropathology and prion replication.

\section{MATERIAL AND METHODS}

\section{BSE challenge of cattle}

Two hundred steers were selected randomly from farms with no history of BSE. These steers were randomly allocated to 2 groups of about 90 individuals in each group. Both groups were housed at the same location, but separately, in the same barn, and managed under the same conditions. The first group remained as unexposed controls; the second group was exposed orally at approximately 6 months of age in August and September 1998, with 100 grams of BSE brain homogenate with a titre of $10^{3.1}$ mouse (i.c./i.p.) units $\mathrm{LD}_{50} / \mathrm{g}$. Infected and control steers slaughtered at similar time points post-infection (PI) and tissues for transcriptome analysis were snap frozen in liquid nitrogen rapidly following slaughter. RNA was extracted from caudal medulla tissue of animals $12,18,40,45$ and 61 months PI in the control group, and in animals at 6,9,12, 42 and 45 months PI in the infected group. The challenged animals were tested for signs of disease by immunohistochemistry test (IHC) analysis of obex and medulla tissues at slaughter. Control animals did not display clinical signs of BSE and IHC test of medulla tissues were negative for $\mathrm{PrP}^{\mathrm{BSE}}$, while infected animals at 42 and $45 \mathrm{PI}$ showed possible pathological signs of BSE (nervousness, hunched posture, hindlimb paresis) and IHC tests were positive for medulla and obex, confirming the BSE status of these animals.

\section{RNA extraction}

Total RNA was extracted using the RNA-Easy Column method (Qiagen, UK) according to the manufacturer instruction. The quality and quantity of RNA was measured using a Bioanalyzer Nano 6000 assay (Agilent, USA).

\section{Microarray hybridization and data analysis}

Samples from controls $(\mathrm{N}=5)$ from different time points post-infection were pooled as were samples from infected $(\mathrm{N}=5)$ animals. The pooling approach was used and as only a few samples were available (Kendziorski et al., 2005) in the present study only one animal was slaughtered at each time point. One microgram of the pooled RNA samples was used for microarray experiments.

Duplicates of 24,000 bovine oligonucleotide probes (www.Bovineoligo.org) representing 16,846 different genes from the bovine genome were spotted onto ultragap slides (Corning, USA) using a Q-array2 spotter (Genetix, UK). One microgram of total RNA was reverse-transcribed using Amino Allyl Message $\mathrm{Amp}^{\mathrm{TM}}$ antisense RNA (aRNA) amplification Kit (Applied Biosystems, USA) and labeled with Cy3 or Cy5 fluorescent dyes (GE Healthcare, USA) according to manufacturer instructions. In total, 4 slides were used to compare control and infected animals, including dye swaps, and two technical replicates (two sets of 24,000 oligonucletide probes). Therefore, each gene was represented 8 times in the statistical analysis. Hybridizations were carried out in a hybridization chamber (Genetix, UK) at $42^{\circ} \mathrm{C}$ 
overnight. Hybridized slides were washed with low stringency buffer (2X SSC and 0.5\% SDS) then high stringency buffer $(0.5 \mathrm{X}$ SSC and $0.2 \%$ SDS $)$ and $0.05 \mathrm{X}$ SSC. Hybridized slides were scanned at 5 micron resolution and their signal intensities were detected by Q-Scan (Genetix, UK).

Data analysis was performed using GeneSifter ${ }^{\mathrm{TM}}$ (VizX Labs, USA). Differences in gene expression levels at different times post-infection were analyzed using the t-test statistical method. Criteria for identifying DE genes were a 2-fold or greater change in expression level, with $\mathrm{P}<0.01$, which was adjusted for multiple testing using Bonferroni correction (Bland and Altman, 1995). The quality filter of $95 \%$ was used to eliminate data from probes in any group that had an intensity variation larger than 5\%. Gene ontology was used to determine the function of DE genes (http://www.geneontology.org).

\section{Quantitative real-time PCR analysis}

The DE genes identified were validated by qRT-PCR using individual samples from both infected and control groups $(\mathrm{N}=10)$. The qRT-PCR analyses of selected genes were performed using amplified RNA due to the very limited amounts of total RNA available. Previous studies have shown that similar values for comparative expression levels are obtained when using either total RNA or aRNA (Taniguchi et al., 2008). The RNA abundance was measured using the TaqMan ${ }^{\circledR}$ Universal PCR Master Mix with gene specific minor grove binder (MGB) probes labeled with FAM and VIC fluorescent dyes (Applied Biosystems, USA). The templates used were 500 ng aRNA obtained by reverse-transcribed synthesis using an aminoallyl kit (Applied Biosystems, USA). The targeted genes, primer and probe sequences for each targeted gene are described in Table 1. Each reaction was carried out in triplicate using StepOnePlus-Real time PCR System (Applied Biosystems, USA). The thermal cycling conditions were as follows: $95^{\circ} \mathrm{C}$ for $20 \mathrm{~s}$ followed by 40 cycles of $95^{\circ} \mathrm{C}$ for $1 \mathrm{~s}$ and $60^{\circ} \mathrm{C}$ for 20 s. Four genes were tested as the endogenous control: GPADH, 18S rRNA, Cyc and RPL12. According to Bestkeeper software (Pfaffl et al., 2004), RPL12 showed the most stable expression in medulla tissues, and therefore was selected as an endogenous control. Cycle threshold $\left(\mathrm{C}_{\mathrm{T}}\right)$ values for each gene were obtained for each sample. Delta $\mathrm{Ct}$ values for each gene were calculated by subtracting the $\mathrm{Ct}$ value of reference gene $\mathrm{Ct}$ from that of the targeted gene. Gene expressions for infected samples were quantitatively measured relative to RNA levels from control animals at a similar time post-infection $(6,12,42$, and 45 months PI). Relative quantification values were determined using the $2^{-\Delta \Delta C \mathrm{CT}}$ method and expressed as fold change in infected versus control animals.

\section{RESULTS AND DISCUSSION}

\section{Microarray analysis of DE genes between control and BSE-infected cattle}

The microarray analysis identified 182 DE genes (133 up-regulated and 49 down-regulated) between caudal medulla tissues of BSE-infected animals and control animals. Among these DE genes 81 genes (67 up-regulated and 14 down-regulated) could be assigned a function according to Gene Ontology (GO) analysis (Table 2). The main categories of GO terms assigned to the DE genes were: cellular process (35 genes), metabolic process (29 genes), 


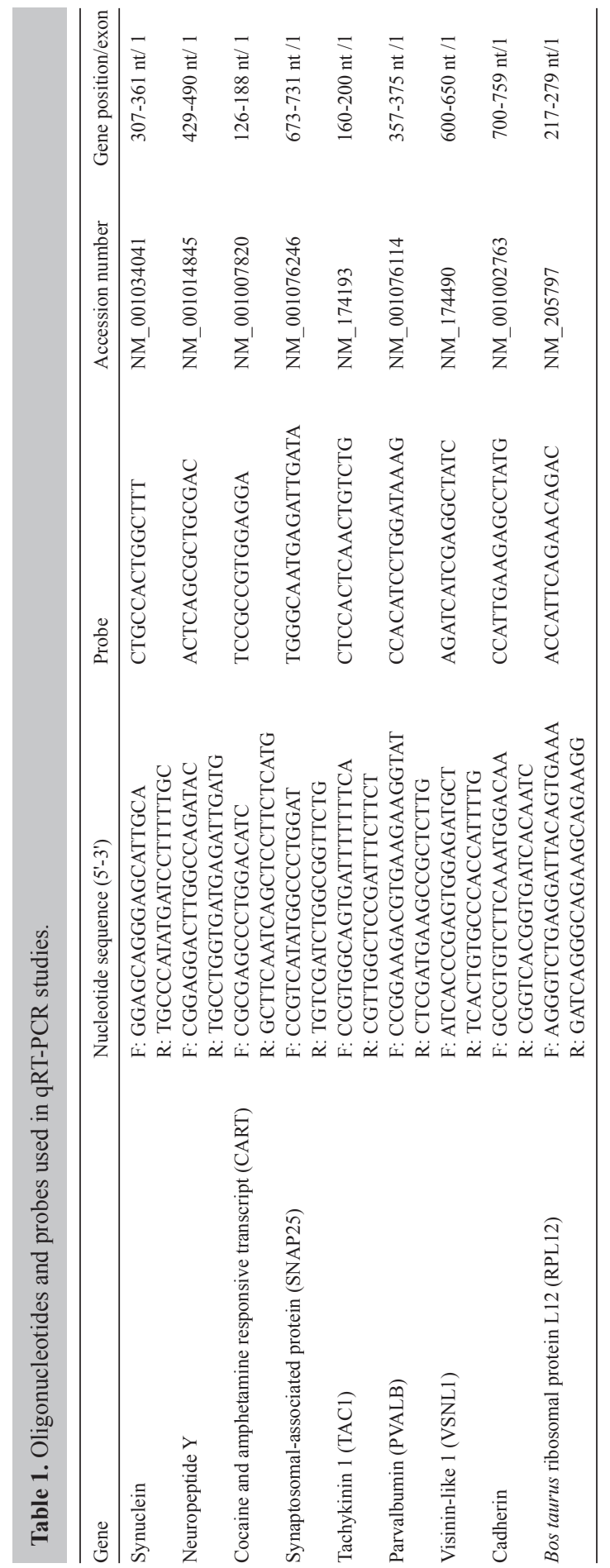


Microarray analysis of DE genes in BSE-infected medulla

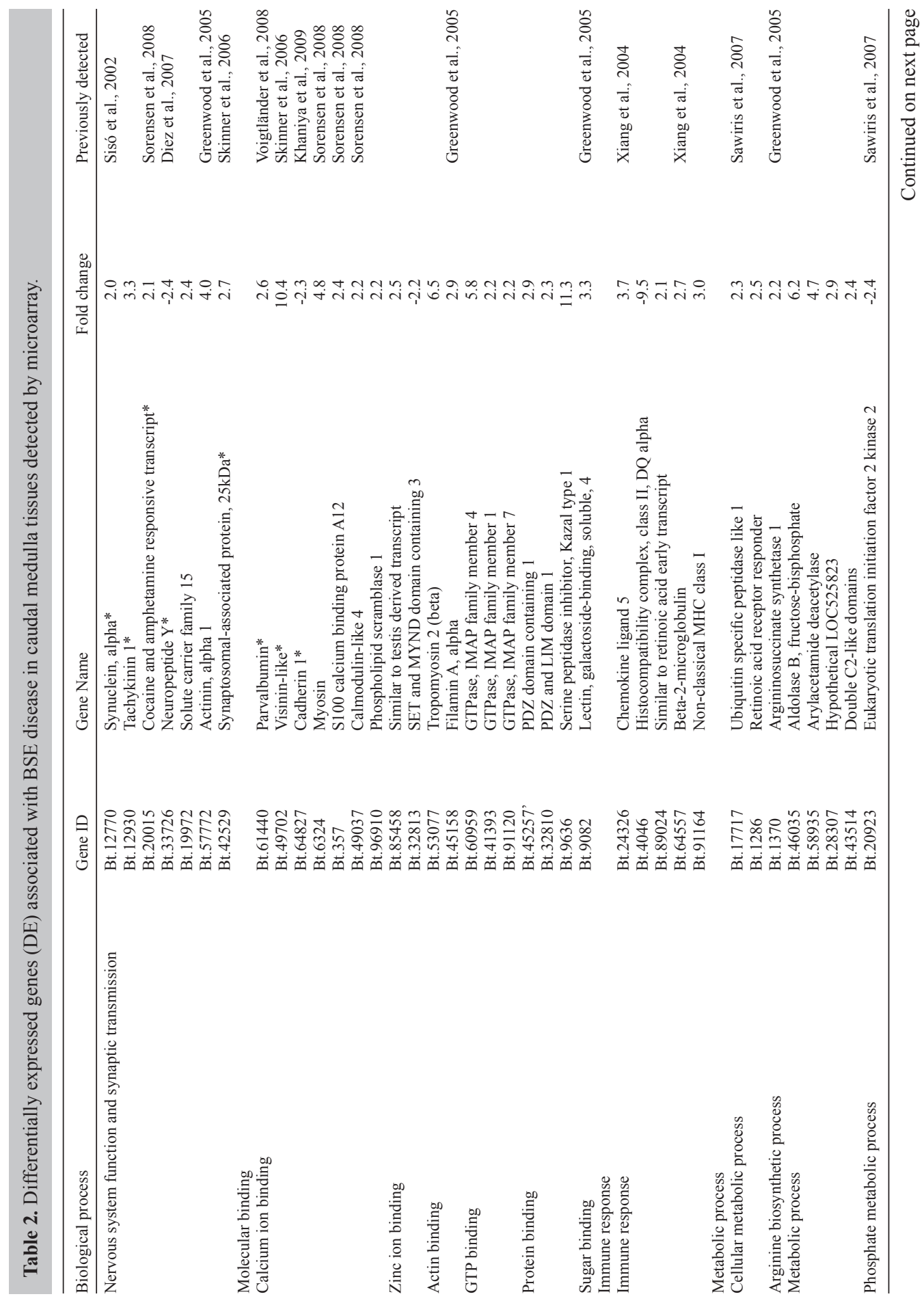

Genetics and Molecular Research 10 (4): 3948-3962 (2011) 


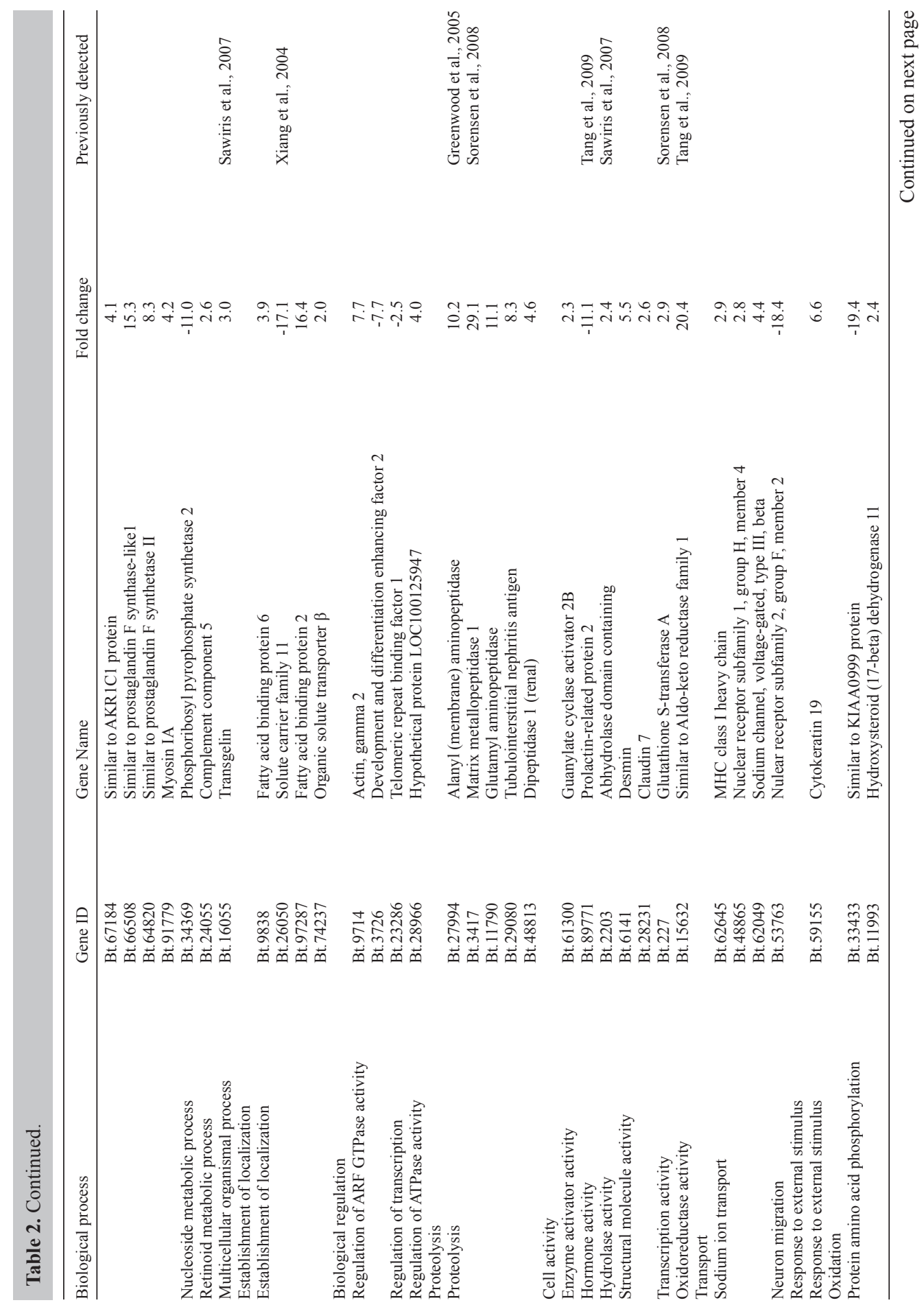




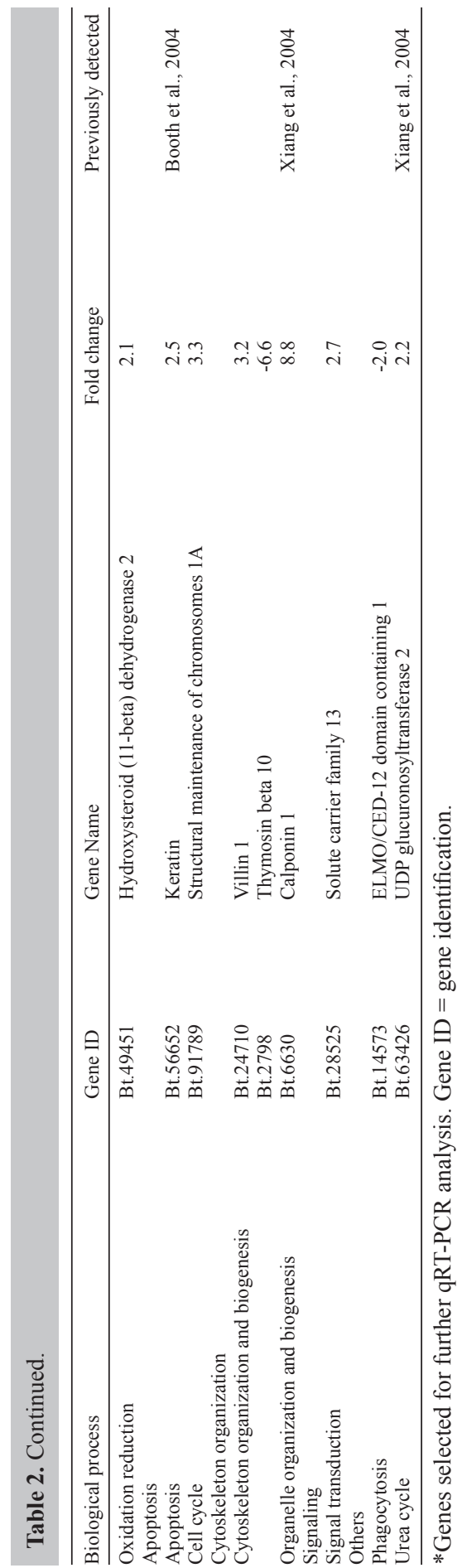


biological regulation (17 genes), regulation of biological process (16 genes), localization (17 genes), establishment of localization (13 genes), response to stimulus (13 genes), developmental process (10 genes) and immune system process ( 9 genes). Many DE genes belong to more than one GO category and for simplicity these genes are presented under biological process functional headings in Table 2. Some DE genes found in this study have been previously reported from the studies of gene expression of TSE progression using mice models, which include: Cocaine and amphetamine responsive transcript, S100 calcium binding, Myosin, Calmodulin, Matrix metalopeptidase, Glutathione S-transferase (Sorensen et al., 2008); Synuclein (Sisó et al., 2002); Parvalbumin (Voigtländer et al., 2008) Neuropeptide Y (Diez et al., 2007); Abhydrolase domain containing, Transgelin, Ubiquitin specific protease (Sawiris et al., 2007); Visinin-like (Skinner et al., 2006); Lectin galactoside-binding, Actinin, Tropomyosin, Arginine succinate synthetase, Alanyl aminopeptidase (Greenwood et al., 2005); $\beta 2$ microglobulin, Solute carrier family 11, UDP-glucuronosyltransferase, Chemokine (Xiang et al., 2004), Synaptosomal associated protein $25 \mathrm{kDa}$, and Keratin (Booth et al., 2004). Many other genes have been found to be differentially expressed in those mice studies and were not detected to be differentially expressed in our analysis in cattle. Besides being different species, the difference in infection method also could be influencing the result. The common method for BSE agent infection in mice is intraperitoneally, while in this study cattle were orally infected. Although gene expression profiling studies in mouse models of BSE infection can provide valuable insights into the possible pathological mechanisms of the disease process, studies in the natural target species (cattle) infected by natural route (oral infection) are crucial for understanding the disease processes.

Recently, Tang et al. (2009) identified 114 DE genes in brains of orally infected cattle vs uninfected controls and $10 \mathrm{DE}$ genes identified by the authors were in common with our analysis. These genes may be an important key in pathogenic processes and need to be better investigated in animals with different genotypes, ages, doses of BSE infection and environment. The common genes detected between our analysis and Tang et al. (2009) were: S100 calcium binding and Calmodulin (up-regulated in both studies); Prolactin-related protein (downregulated in both studies); GTPase, IMAP family member, Histocompatibility complex, class II, Metallopeptidase (variable gene expression levels in the first study and up-regulated in our analyses) and Myosin, Glutathione S transferase A, Aldo-Keto reductase family and Nuclear receptor subfamily group $\mathrm{H}$ (down-regulated in the first study and up-regulated in our analysis). Notwithstanding these 10 genes that are in common between the studies, many genes identified were specific to one or the other study, which most likely is because different brain regions were used in the two studies: medulla was used in the present study while brain stem was examined by Tang et al. (2009). Other factors that may have influenced the difference in results include the variation in the genetic composition of the animals; different microarray platforms and experimental conditions. In the last decade many studies have identified a large number of genes that are DE between control and TSE infected animals; however, only a few have been shown to contribute directly to prion pathogenesis (Tamgüney et al., 2008).

The DE genes identified in the present study were mapped to biochemical pathways by the Kyoto Encyclopedia of Genes and Genomes (KEGG) database. In total, 13 DE genes were found to be associated with 26 different networks including: Neuroactive ligand-receptor interaction; MAPK signaling pathway; cell adhesion molecules (CAMs); cell communication; cell cycle; focal adhesion; PPAR signaling pathway; SNARE interactions in vesicular 
transport; and regulation of actin cytoskeleton, among others (Table 3). Further work will be necessary to link these changes in expression to the biological functions involved in the development of BSE disease.

Table 3. KEGG pathway analysis of DE genes detected by microarray.

\begin{tabular}{|c|c|}
\hline KEGG pathway analysis & Gene identified using microarray \\
\hline Adherens junction & $\begin{array}{l}\text { Actin, gamma 2, smooth muscle, enteric } \\
\text { Cadherin } 1 \text {, type } 1 \text {, E-cadherin (epithelial) }\end{array}$ \\
\hline Androgen and estrogen metabolism & Hydroxysteroid (11-beta) dehydrogenase 2 \\
\hline Focal adhesion & Actin, gamma 2, smooth muscle, enteric \\
\hline Filamin A, alpha (actin binding protein 280) & \\
\hline $\begin{array}{l}\text { Metabolism of xenobiotics by cytochrome P } 450 \\
\text { UDP glucuronosyltransferase } 2 \text { family, polypeptide B10 }\end{array}$ & Cytochrome P450, subfamily IIIA, polypeptide 4 \\
\hline PPAR signaling pathway & $\begin{array}{l}\text { Fatty acid binding protein } 6 \text {, ileal (gastrotropin) } \\
\text { Matrix metallopeptidase } 1 \text { (interstitial collagenase) }\end{array}$ \\
\hline Neuroactive ligand-receptor interaction & Tachykinin 1 \\
\hline SNARE interactions in vesicular transport & Synaptosomal-associated protein, $25 \mathrm{kD}$ \\
\hline MAPK signaling pathway & Filamin A, alpha (actin binding protein 280) \\
\hline C21-Steroid hormone & Hydroxysteroid (11-beta) dehydrogenase 2 \\
\hline Cell adhesion molecules (CAMs) & Cadherin 1, type 1, E-cadherin (epithelial) \\
\hline Cell cycle & gamma-Hexachlorocyclohexane degradation \\
\hline Cytochrome P450, subfamily IIIA, polypeptide 4 & \\
\hline Glutathione metabolism & $\begin{array}{l}\text { Alanyl (membrane) aminopeptidase (aminopeptidase } \mathrm{N} \text {, } \\
\text { aminopeptidase } \mathrm{M} \text {, microsomal }\end{array}$ \\
\hline Hematopoietic cell lineage & $\begin{array}{l}\text { Alanyl (membrane) aminopeptidase (aminopeptidase } \mathrm{N} \text {, } \\
\text { aminopeptidase } \mathrm{M} \text {, microsomal }\end{array}$ \\
\hline Leukocyte transendothelial migration & Actin, gamma 2 , smooth muscle, enteric \\
\hline Linoleic acid metabolism & Cytochrome P450, subfamily IIIA, polypeptide \\
\hline Melanoma & Cadherin 1, type 1, E-cadherin (epithelial) \\
\hline Pentose and glucuronate interconversions & UDP glucuronosyltransferase 2 family, polypeptide B10 \\
\hline Porphyrin and chlorophyll metabolism & UDP glucuronosyltransferase 2 family, polypeptide B10 \\
\hline Regulation of actin cytoskeleton & Actin, gamma 2 , smooth muscle, enteric \\
\hline Renin-angiotensin system & $\begin{array}{l}\text { Alanyl (membrane) aminopeptidase (aminopeptidase } \mathrm{N} \text {, } \\
\text { aminopeptidase } \mathrm{M} \text {, microsomal }\end{array}$ \\
\hline Glutamyl aminopeptidase (aminopeptidase A) & \\
\hline Starch and sucrose metabolism & UDP glucuronosyltransferase 2 family, polypeptide B10 \\
\hline Thyroid cancer & Cadherin 1, type 1, E-cadherin (epithelial) \\
\hline Tight junction & Actin, gamma 2 , smooth muscle, enteric \\
\hline
\end{tabular}

\section{Functional classification of DE genes}

Synapse loss has been identified as an early and critical pathophysiological event in neurodegenerative diseases. Electron microscopic studies have shown that degeneration of synapses precedes neuronal degeneration in scrapie-infected murine hippocampus (Jeffrey et al., 2000). In addition to synapse loss, the calcium homeostasis network has also been reported to be associated with prion diseases by various researchers (Greenwood et al., 2005; Sorensen et al., 2008). Therefore, in the present study the DE genes associated with these two networks were validated by qRT-PCR (Figure 1).

\section{Degeneration of synapses associated DE genes in BSE-infected medulla tissues}

For a better understanding of the molecular events involved in synapses degeneration during disease progression in BSE-infected cattle, five genes were selected for qRT-PCR analysis at different times post-infection. Three genes (Tachykinin, Synuclein, Cocaine and 


\section{DE Genes}

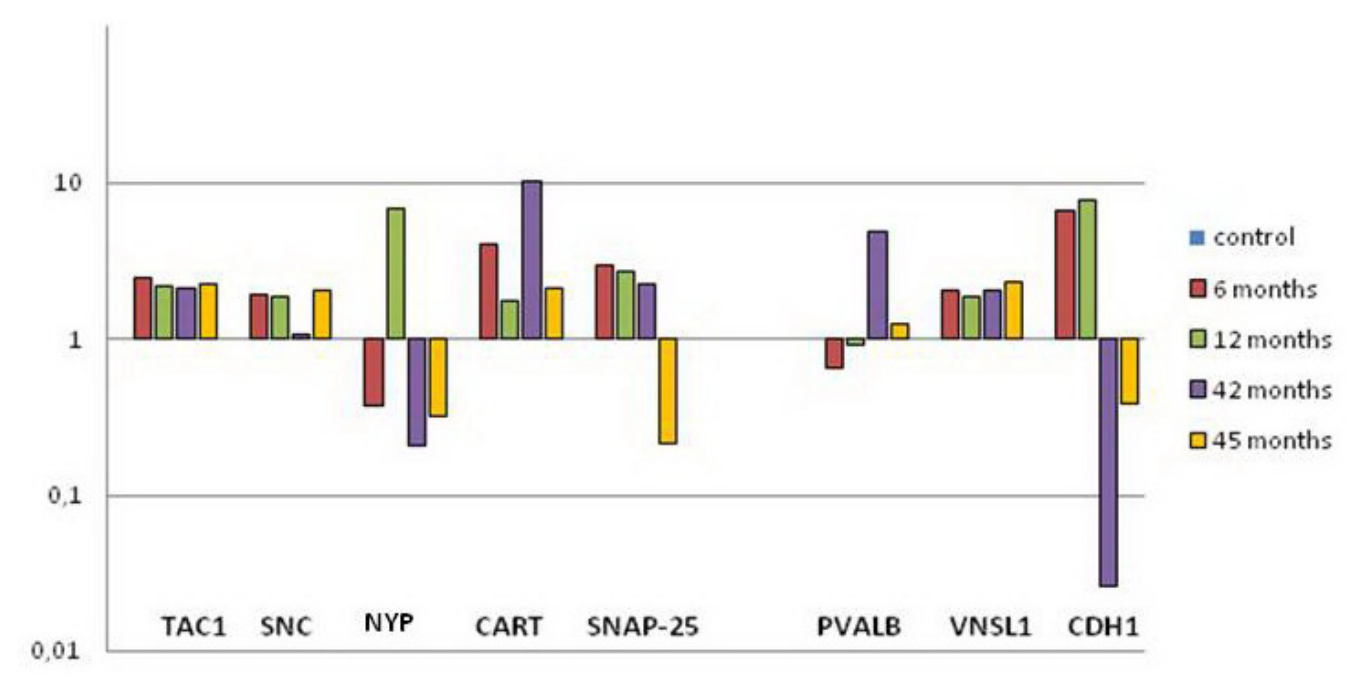

Figure 1. Quantitative real-time PCR analysis of DE genes: tachykinin (TACH1); synuclein (SNC); neuropeptide Y (NPY), cocaine amphetamine-responsive transcript (CART) and synaptosomal-associated protein $25 \mathrm{kDa}$ (SNAP-25), parvalbumin (PVALB), visinin-like (VNSL1) and cadherin 1 (CDH1). Gene expression was compared between control animals and 100g infected animals at 6,12, 42 and 45 months post-infection. Gene expressions of infected animals were quantitatively measured relative to RNA levels from control animals.

amphetamine responsive transcript) were found to be up-regulated in all time points from 6 to 45 months post-infection; the synaptosomal associated protein $25 \mathrm{kDa}$ was up-regulated in almost all times post-infection except 45 months PI; while Neuropeptide Y was down-regulated in almost all times post-infection except at 12 months PI.

The biological functions of some of the DE genes identified in this study and also in previous studies were investigated further. CART codes for a peptide that has been implicated in a variety of brain functions, including the protection offered by estradiol against ischemic brain injury in stroke and other neurodegenerative diseases (Xu et al., 2006). CART was found to be 2-fold overexpressed in pooled samples from infected animals vs. the control pool. This upregulation associated with infection was validated by qRT-PCR at 6, 12, 42 and 45 months PI with a 3.9-, 1.7-, 10.0- and 2.0-fold change respectively. A recent publication by Murdoch et al. (2010) using sib-TDT analysis identified two significant SNPs on BTA 20 associated with BSE incidence within the CART gene. These data suggest that there may be a genetic effect controlling susceptibility to BSE which may be mediated through the expression level of CART, which may also be a signature of the BSE infection. In addition, CART has previously been found to be DE expressed in the brain of mice infected with scrapie (Sorensen et al., 2008) and interestingly in Peyer's patches of BSE-infected cattle (Khaniya et al., 2009). These observations further suggest a role of this gene in the disease process.

Tachykinin (TAC1) is a neurotransmitter in the central and peripheral nervous system (Graham et al., 2004), smooth muscle (Savi et al., 1992), as well as being involved in immunologic and inflammatory processes (Brain, 1997). In the present study, TAC1 was found to be overexpressed in the medulla of infected animals (3.3 fold in microarray study and 2.4, 
2.1, 2.0 and 2.2 at $6,12,42$ and 45 months respectively PI by qRT-PCR). The variation in expression of TAC1 in response to prion disease was not previously detected. Further studies will therefore be necessary to investigate if changes in TAC1 expression are associated with various TSE infection models.

Presence of amyloid fibrils is considered to be a signature of the neurodegeneration in some TSEs although the mechanism behind the cellular degeneration associated with fibril formation remains unknown (Bhak et al., 2009). $\alpha$-Synuclein (SNC) is an amyloidogenic protein with presynaptic and chaperone-like activity (Haik et al., 2002). This protein is associated with Parkinson's disease and contributes to the formation of intracellular Lewy body and its radiating filamentous structures (Bhak et al., 2009). SNC has also been associated with prion protein accumulation and neurodegeneration in a hamster scrapie model (Haik et al., 2002). In this study, SNC showed increased expression in samples from all infected animals, both in the pooled infected and control samples analyzed by microarray ( 2.0 fold) and by qRT-PCR, showing a 1.9-, 1.8-, 1.0- and 2.0-fold change at 6, 12, 42 and 45 months PI.

Neuropeptide Y (NPY) is a neurotransmitter known to be associated with a number of physiologic processes in the brain. NPY has been shown to be overexpressed in a number of TSE experimental mice models (Diez et al., 2007). According to Diez et al. (2007), the overexpression of this gene could reflect a protective and/or regenerative response counteracting neuronal degeneration resulting from cerebral nerve injury caused by $\operatorname{PrP}^{\mathrm{TSE}}$ infection. However, in contrast to previous studies expression of NYP was found to decrease in infected animals (-2.4-fold change in the pooled samples) which was validated by the qRT-PCR which showed a $0.3-, 0.2$ - and 0.3 -fold change at 6,42 and 45 months PI, respectively.

SNAP-25 is involved in the regulation of neurotransmitter vesicle trafficking (Sollner et al., 1993), and its expression is widely distributed in the brain. Reduced SNAP-25 expression has been reported in the brain of scrapie infected mice (Sisó et al., 2002). In the present analyses, the expression of SNAP-25 was up-regulated at 6, 12 and 42 months PI (2.9-, 2.6and 2.2-fold change respectively). However, in late stages of the disease (45 months PI), the expression of SNAP-25 was reduced (to 0.7 fold of the control value). Down-regulation of the SNAP-25 gene and dysfunction of neurotransmitter vesicle trafficking may be hypothesized in late stages of the disease with associated loss of synapse function in the medulla.

The differences of expression patterns of NPY, CART, TAC1 and SNAP-25 associated with BSE infection of cattle reported here compared with results from studies in mice suggest that responses to prion infection may differ between mice and cattle. These differences in gene expression patterns between experimental models could also be due to individual biological variation, differences in experimental design and route of infection.

\section{Calcium homeostasis associated DE genes in BSE-infected medulla tissues}

Changes in calcium homeostasis have been associated with prion disease (Greenwood et al., 2005; Sorensen et al., 2008) and other neurodegenerative disorders, such as Down's syndrome and Parkinson's disease (Muramatsu et al., 2003). The microarray of pooled infected samples compared with control samples identified a down-regulation of seven genes associated with calcium homeostasis (Table 2). Among these genes encoding Parvalbumin (PVALB), Visinin-like 1(VSNL1) and Cadherin 1 were selected for qRT-PCR analysis.

PVALB is a calcium binding albumin protein present mainly in GABAergic inter-neu- 
rons of the nervous system that have been shown to influence the survival or death of neurons (Voigtländer et al., 2008). PVALB was found to be over-expressed in the caudal medulla of BSE-infected animals compared with controls (2.6 fold comparing pooled infected samples with control samples and 4.7 and 1.2 fold, respectively by qRT-PCR at 42 and 45 months PI) and down-expressed in early stage of disease (6 and 12 months PI). VSNL1 is a member of neuronal $\mathrm{Ca}^{2+}$ sensor protein which modulates $\mathrm{Ca}^{2+}$ dependent cell signaling; however, its function in other tissues remains largely unknown (Dai et al., 2006). VSNL1 was also found be down-regulated in brain tissues of scrapie infected mice (Skinner et al., 2006); however, in the present study, VSNL-1 was found to be up-regulated in both pooled samples (10.4 fold in infected $v s$ controls) and qRT-PCR analyses $(2.0 ; 1.8,2.0$ and 2.3 fold at $6,12,42$ and 45 months PI respectively). CDH1 is a calcium dependent cell-cell adhesion membrane glycoprotein that plays an important role in adherens junctions between epithelial cells and downregulating cell growth (Nelson, 2008). CDH1 is an important cell surface receptor of Schwann cells, and plays a pivotal role in the maintenance and regeneration of axons in the peripheral nervous system (Bhatheja and Field, 2006). This protein was found to be up-regulated at 6 and 12 months PI (6.6 and 7.7 fold, respectively) and down-regulated at 42 and 45 months PI (0.02 and 0.3 fold, respectively). Altered expression of $\mathrm{CDH} 1$ has not been reported previously from other TSE infected brain tissues. However, Khaniya et al. (2009) detected over-expression of CDH1 in Peyer's patch tissue of BSE-infected cattle, suggesting that it may be involved in BSE progression.

\section{CONCLUSIONS}

A better understanding of the molecular mechanism involved in BSE infection will hopefully shed some light on the mechanisms of TSE disease progression. This study identified genes that are differentially expressed in caudal medulla from BSE-infected $v s$ control animals using microarray analysis. Many of the DE genes identified here have also been reported in mice models, revealing some common mechanisms associated with prion pathogenesis among different species. DE genes associated with two different biological functions (synapse function and calcium homeostasis) were validated by qRT-PCR, showing that expression patterns vary during the time course of the disease. However the study reported here used a small number of samples and additional studies are required using a larger number of animals at each time point to confirm the general validity of these results It would also be appropriate to examine the expression of these in different environments. The identification of variations in gene expression in response to BSE infection may help to understand the disease process and also provide indications of target genes and proteins that could be used in diagnosing infection or in treatment and prevention of TSE diseases.

\section{ACKNOWLEDGMENTS}

We would like to thank the Veterinary Laboratories Agency (Weybridge, UK) for the tissue samples that were used for the preparation of the RNA used in this study (funded by Defra project SE1736 and EU project FAIR CT98-778). Research supported by PrionNet Canada, Alberta Prion Institute and Alberta Bovine Genomic Program. JLW thanks the Cariplo Foundation for their support. 


\section{REFERENCES}

Aguzzi A, Baumann F and Bremer J (2008). The prion's elusive reason for being. Annu. Rev. Neurosci. 31: 439-477.

Almeida LM, Basu U, Khaniya B, Taniguchi M, et al. (2011). Gene expression in the medulla following oral infection of cattle with bovine spongiform encephalopathy. J. Toxicol. Environ. Health A. 74: 110-126.

Bhak G, Choe YJ and Paik SR (2009). Mechanism of amyloidogenesis: nucleation-dependent fibrillation versus doubleconcerted fibrillation. BMB Rep. 42: 541-551.

Bhatheja K and Field J (2006). Schwann cells: origins and role in axonal maintenance and regeneration. Int. J. Biochem. Cell Biol. 38: 1995-1999.

Bland JM and Altman DG (1995). Multiple significance tests: the Bonferroni method. BMJ 310: 170.

Booth S, Bowman C, Baumgartner R, Sorensen G, et al. (2004). Identification of central nervous system genes involved in the host response to the scrapie agent during preclinical and clinical infection. J. Gen. Virol. 85: 3459-3471.

Brain SD (1997). Sensory neuropeptides: their role in inflammation and wound healing. Immunopharmacology 37: 133152.

Dai FF, Zhang Y, Kang Y, Wang Q, et al. (2006). The neuronal $\mathrm{Ca}^{2+}$ sensor protein-1 is expressed in pancreatic islet and regulates insulin secretion. J. Biol. Chem. 281: 21942-21953.

Dandoy-Dron F, Guillo F, Benboudjema L, Deslys JP, et al. (1998). Gene expression in scrapie. Cloning of a new scrapieresponsive gene and the identification of increased levels of seven other mRNA transcripts. J. Biol. Chem. 273: 7691-7697.

Diedrich JF, Minnigan H, Carp RI, Whitaker JN, et al. (1991). Neuropathological changes in scrapie and Alzheimer's disease are associated with increased expression of apolipoprotein $\mathrm{E}$ and cathepsin D in astrocytes. J. Virol. 65: 4759-4768.

Diez M, Groth D, DeArmond SJ, Prusiner SB, et al. (2007). Changes in neuropeptide expression in mice infected with prions. Neurobiol. Aging 28: 748-765.

Graham GJ, Stevens JM, Page NM, Grant AD, et al. (2004). Tachykinins regulate the function of platelets. Blood 104: 1058-1065.

Greenwood AD, Horsch M, Stengel A, Vorberg I, et al. (2005). Cell line dependent RNA expression profiles of prioninfected mouse neuronal cells. J. Mol. Biol. 349: 487-500.

Haik S, Privat N, Adjou KT, Sazdovitch V, et al. (2002). Alpha-synuclein-immunoreactive deposits in human and animal prion diseases. Acta Neuropathol. 103: 516-520.

Hernandez-Sanchez J, Waddington D, Wiener P, Haley CS, et al. (2002). Genome-wide search for markers associated with bovine spongiform encephalopathy. Mamm. Genome 13: 164-168.

Jeffrey M, Halliday WG, Bell J, Johnston AR, et al. (2000). Synapse loss associated with abnormal PrP precedes neuronal degeneration in the scrapie-infected murine hippocampus. Neuropathol. Appl. Neurobiol. 26: 41-54.

Juling K, Schwarzenbacher H, Williams JL and Fries R (2006). A major genetic component of BSE susceptibility. BMC Biol. 4: 33.

Kendziorski C, Irizarry RA, Chen KS, Haag JD, et al. (2005). On the utility of pooling biological samples in microarray experiments. Proc. Natl. Acad. Sci. U. S. A. 102: 4252-4257.

Khaniya B, Almeida L, Basu U, Taniguchi M, et al. (2009). Microarray analysis of differentially expressed genes from Peyer's patches of cattle orally challenged with bovine spongiform encephalopathy. J. Toxicol. Environ. Health A 72: 1008-1013.

Kopacek J, Sakaguchi S, Shigematsu K, Nishida N, et al. (2000). Upregulation of the genes encoding lysosomal hydrolases, a perforin-like protein, and peroxidases in the brains of mice affected with an experimental prion disease. J. Virol. 74: 411-417.

Moreno CR, Lantier F, Lantier I, Sarradin P, et al. (2003). Detection of new quantitative trait Loci for susceptibility to transmissible spongiform encephalopathies in mice. Genetics 165: 2085-2091.

Muramatsu Y, Kurosaki R, Watanabe H, Michimata M, et al. (2003). Cerebral alterations in a MPTP-mouse model of Parkinson's disease - an immunocytochemical study. J. Neural Transm. 110: 1129-1144.

Murdoch BM, Clawson ML, Laegreid WW, Stothard P, et al. (2010). A 2cM genome-wide scan of European Holstein cattle affected by classical BSE. BMC Genet. 11: 20.

Nelson WJ (2008). Regulation of cell-cell adhesion by the cadherin-catenin complex. Biochem. Soc. Trans. 36: 149-155.

Pfaffl MW, Tichopad A, Prgomet C and Neuvians TP (2004). Determination of stable housekeeping genes, differentially regulated target genes and sample integrity: BestKeeper-Excel-based tool using pair-wise correlations. Biotechnol. Lett. 26: 509-515.

Prusiner SB and Scott MR (1997). Genetics of prions. Annu. Rev. Genet. 31: 139-175. 
Riemer C, Neidhold S, Burwinkel M, Schwarz A, et al. (2004). Gene expression profiling of scrapie-infected brain tissue. Biochem. Biophys. Res. Commun. 323: 556-564.

Savi P, Laplace MC, Le FG, Emonds-Alt X, et al. (1992). The NK1 receptor is involved in the neurokinin-induced shape change of rabbit platelets. FEBS Lett. 312: 200-202.

Sawiris GP, Becker KG, Elliott EJ, Moulden R, et al. (2007). Molecular analysis of bovine spongiform encephalopathy infection by cDNA arrays. J. Gen. Virol. 88: 1356-1362.

Sisó S, Puig B, Varea R, Vidal E, et al. (2002). Abnormal synaptic protein expression and cell death in murine scrapie. Acta Neuropathol. 103: 615-626.

Skinner PJ, Abbassi H, Chesebro B, Race RE, et al. (2006). Gene expression alterations in brains of mice infected with three strains of scrapie. BMC Genom. 7: 114.

Sollner T, Whiteheart SW, Brunner M, Erdjument-Bromage H, et al. (1993). SNAP receptors implicated in vesicle targeting and fusion. Nature 362: 318-324.

Sorensen G, Medina S, Parchaliuk D, Phillipson C, et al. (2008). Comprehensive transcriptional profiling of prion infection in mouse models reveals networks of responsive genes. BMC Genom. 9: 114.

Tamgüney G, Giles K, Glidden DV, Lessard P, et al. (2008). Genes contributing to prion pathogenesis. J. Gen. Virol. 89: 1777-1788.

Tang Y, Xiang W, Hawkins SA, Kretzschmar HA, et al. (2009). Transcriptional changes in the brains of cattle orally infected with the bovine spongiform encephalopathy agent precede detection of infectivity. J. Virol. 83: 9464-9473.

Taniguchi M, Guan LL, Zhang B, Dodson MV, et al. (2008). Adipogenesis of bovine perimuscular preadipocytes. Biochem. Biophys. Res. Commun. 366: 54-59.

Voigtländer T, Unterberger U, Guentchev M, Schwaller B, et al. (2008). The role of parvalbumin and calbindin D28k in experimental scrapie. Neuropathol. Appl. Neurobiol. 34: 435-445.

Xiang W, Windl O, Wunsch G, Dugas M, et al. (2004). Identification of differentially expressed genes in scrapie-infected mouse brains by using global gene expression technology. J. Virol. 78: 11051-11060.

$\mathrm{Xu}$ Y, Zhang W, Klaus J, Young J, et al. (2006). Role of cocaine- and amphetamine-regulated transcript in estradiolmediated neuroprotection. Proc. Natl. Acad. Sci. U. S. A. 103: 14489-14494.

Zhang C, De Koning DJ, Hernandez-Sanchez J, Haley CS, et al. (2004). Mapping of multiple quantitative trait loci affecting bovine spongiform encephalopathy. Genetics 167: 1863-1872. 